\title{
Numerical Simulation of High Level Sound Generated in Boiler tube Bank Duct
}

\author{
Masaaki Mori, Takayuki Masumoto, Kunihiko Ishihara
}

\begin{abstract}
Heat exchangers and boilers are widely used in various plants such as power plants and chemical plants. In heat exchangers and boilers, tube banks are set in a duct such that water passes through inside the tubes and warm gas outside the tubes. Due to the external flow around tube banks, the resonance phenomenon called the self-sustained tone occurs at a certain velocity. The self-sustained tone might cause noise problems in the surroundings, cause losses due to plant shutdown, etc. The self-sustained tone has been studied, and the generation mechanism has been experimentally and numerically clarified. In this paper, we have performed numerical simulations of high level sound generated in boiler tube bank duct with the flow and an acoustic simulation without the flow for a practical generation prediction of a self-sustained tone. The results show a good agreement with measurement data, and that acoustic resonance phenomenon has been numerically captured and clarified. There is the slight difference in the resonance frequencies obtained from the acoustic simulation (without the flow) by means of BEM and the CFD simulation or the experiments due to the absence or presence of the flow.
\end{abstract}

Index Terms - Self-Sustained Lock-in, Resonance, CFD and BEM.

\section{INTRODUCTION}

In heat exchangers, such as boilers and gas heaters, tube banks are set in a duct and the water passes through inside the tube and warm gas outside the tube. When the flow velocity increases Karman vortex shedding occurs behind the tubes. The vortex shedding frequency is proportional to the velocity. On the other hand, the duct has an acoustic natural frequency determined by the duct size and the sound speed. The acoustic natural frequency is independent of the flow velocity. A resonance phenomenon occurs at a certain velocity when the vortex shedding and acoustic natural frequencies coincide [1-15]. When the flow velocity increases, the vortex shedding frequency suddenly locks on to the acoustic natural frequency of the duct. This is a lock-in (lock-on) phenomenon. Fig. 1 shows the relation between frequency and velocity in a Lock-in phenomenon. When a lock-in phenomenon occurs, a high level of sound is generated and its generation mechanism is called "self-sustained tone" $[13,15]$. The self-sustained tone might cause noise problems, and also cause plant shutdown and hence production losses, etc. In a lock-in phenomenon, as shown in Fig. 1, the frequency slightly rises as the flow velocity increases. Furthermore, when the acoustic

Masaaki Mori, Mechanical CAE Division, CYBERNET SYSTEMS CO.,LTD., 3 Kanda-neribeicho, Chyoda-ku, Tokyo, Japan, Phone+81352973767.

Takayuki Masumoto, Mechanical CAE Division, CYBERNET SYSTEMS CO.,LTD., 3 Kanda-neribeicho, Chyoda-ku, Tokyo, Japan, Phone+81352973767

Kunihiko Ishihara, Department of Health and Welfare, Tokushima Bunri University, Shido, Sanuki-city, Kagawa, Japan, +81878945111 damping of the sound field in the duct is large, the lock-in phenomenon occurs at a certain flow velocity and does not occur thereafter as the flow velocity increases. However, when the acoustic damping is small, a high level sound continues with increasing flow velocity, and the sound pressure level remains substantially constant [15]. This is due to the fact that when the shedding frequency of the karman vortex generated as the gas passes through the tube bank and the acoustic resonance frequency of the duct are close to each other, the interior sound field in the duct is strongly excited and the strong vortices those has a shedding frequency matching the resonance frequency in the duct width direction are generated. As a result, the sound field is further excited in the duct. It is known that this phenomenon is caused by a self-excited mechanism. Also, focused on the self-excited acoustic resonance of two side-by-side cylinders in a duct, the mechanism of the self-excited acoustic resonance is investigated by experiments and numerical solutions [16, 17]. They have found that dynamic lift fluctuation on the cylinders and strong in-phase vortex shedding synchronization are generated by the acoustic resonance. Thus, the self-sustained tone has been studied and the generation mechanism has been experimentally and numerically clarified [1-15, 16-17].

The purpose in this paper is to capture and predict the generation of the self-sustained tone of tube arrays in a duct numerically using commercial CFD and an acoustic BEM simulation codes. We perform compressible CFD and acoustic BEM simulations to capture the self-sustained tone and acoustic resonance frequencies, and compare the simulation results with the measurements [15].

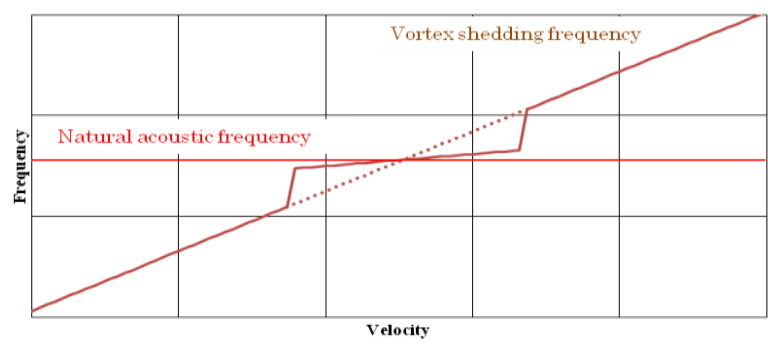

Fig. 1 Lock-in phenomenon

\section{EXPERIMENTAL SETUP, MEASUREMENTS AND RESULTS}

\section{A. Experimental Setup}

The experimental setup is shown in Fig. 2 (a). The size of the duct is $1420 \mathrm{~mm}$ in length, $200 \mathrm{~mm}$ in height, and $234 \mathrm{~mm}$ in width. The tube bank is set as shown in Fig. 2 (a). The microphone is put near the duct outlet as shown in Fig. 2 (a). The flow is driven through the duct by a blower and the flow rate can be controlled by an inverter. The tube bank consists of an array of bronze tubes of diameter $\mathrm{D}=6 \mathrm{~mm}$. The array geometry is a square with spacing $\mathrm{T} / \mathrm{D}=2.0$ and $\mathrm{L} / \mathrm{D}=2.0$, 
where $\mathrm{T}$ is the transverse center-to-center spacing, $\mathrm{L}$ is the longitudinal center to center spacing and $\mathrm{D}$ is the tube outside diameter as shown in Fig. 2 (b). The tube bank consists of 9 rows of tubes in the flow direction and 19 tubes in the direction perpendicular to the flow, and the length in the flow direction is $102 \mathrm{~mm}$. The flow velocity in the duct is measured by using a hot wire anemometer and the sound pressure level of the self-sustained tone is measured using the sound level meter. The blower has a maximum air flow rate of 100 $\mathrm{m} 3 / \mathrm{min}$, a maximum static pressure of $930 \mathrm{~Pa}$, and a motor output of $3.7 \mathrm{~kW}$. The rotational speed is controlled by the inverter to change the flow velocity. An air is supplied to the duct by the blower and the air flow passes through the tube bank. The vortex shedding occurs in and behind the tube bank The vortex shedding frequency is proportional to the flow velocity. When it is close to the acoustic natural frequency of the duct system, then a high level sound occurs.

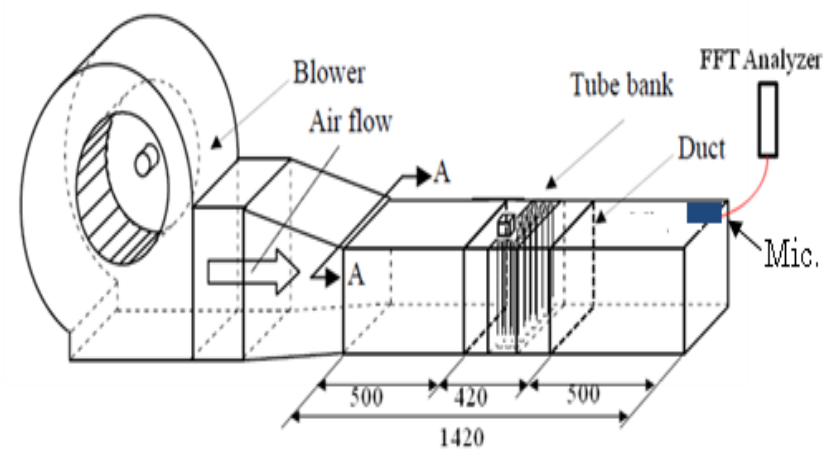

(a)

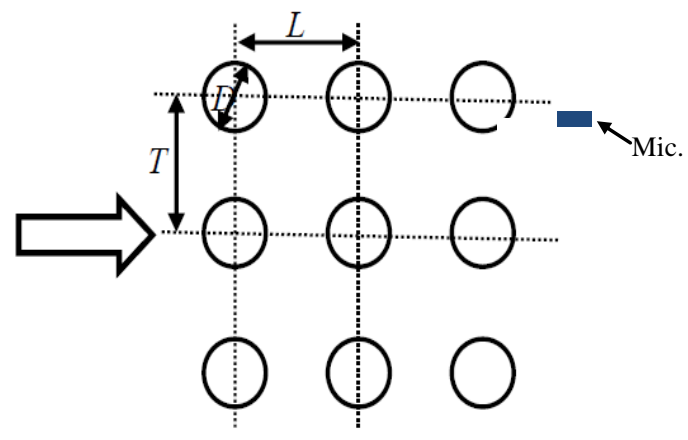

(b)

Fig. 2 (a) Experimental Setup; (b) Array geometry of tube bank.

\section{B. Velocity Measurements}

A flow velocity measurement hole was provided at a position $125 \mathrm{~mm}$ upstream of the tube group, and the flow velocity was also measured there $(U)$. The following data uses the flow velocity $U$. The gap flow velocity is the flow velocity between the pipe and pipe, and the ratio of the area of the duct outlet to the area of the tube bank clearance is 1.95 , hence the gap flow velocity $V_{g}$ is obtained from the continuous equation, and results in $V_{g}=1.95 U$. As the operation of the blower, the inverter frequency finv was varied within the range of 20 to $60 \mathrm{~Hz}$.

\section{Sound Pressure Measurements}

We observe changes in sound pressure level and sound pressure spectrum due to an increase in flow velocity. The sound level meter was installed near the duct outlet shown in Fig. 2(a) using NL-20 manufactured by Rion. The C characteristic was used for measurement. Sound pressure signals are measured and converted to frequency domain with FFT Analyzer. The frequency range is from $200 \mathrm{~Hz}$ to 2000 $\mathrm{Hz}$, the sampling frequency is $10000 \mathrm{~Hz}$, the number of averages is 1000, and the frequency resolution is $20 \mathrm{~Hz}$.

\section{Experimental Results}

Fig. 3 shows the sound pressure spectrum at each inverter frequency. Inverter frequencies are $30,40,46$ and $50 \mathrm{~Hz}$, and corresponds to gap velocities $11.4,15.7,19.6$ and $21.3 \mathrm{~m} / \mathrm{s}$, respectively. As shown in Fig. 3, the self-sustained tone slightly appears at finv $=46 \mathrm{~Hz}$, and clearly appears at finv $=$ $50 \mathrm{~Hz}$. This peak frequency is $740 \mathrm{~Hz}$. Fig. 4 shows the relation between overall sound pressure level and the gap velocity. The sound pressure level increases with increasing the gap velocity, and follows the 5th power law when the self-sustained tone is not generated (when the gap velocity is low). However, the sound pressure level suddenly increases when the self-sustained tone is generated (points surrounded by the red circle). As shown in Fig. 4, the overall sound pressure level saturates, and remains high when the gap flow velocity is over $22 \mathrm{~m} / \mathrm{s}$ [15]. In this experiment, the self-excited tone is generated when the gap velocity is over around $20 \mathrm{~m} / \mathrm{s}$. Therefore, if the Strouhal number St is 0.22 , the vortex shedding frequency $f_{v}$ is $f_{v}=S t^{*} V_{g} / D=0.22$ $* 20 / 0.006=733 \mathrm{~Hz}$. Meanwhile, the resonance frequency $f_{a}$ in the width direction of the duct is $f_{a}=c_{e} / 2 L=$ $337.5531 / 2 / 0.234=721.26 \mathrm{~Hz}$.

Considering the coupled mode in the longitudinal direction, the resonance frequency $f_{a}$ is $730.99 \mathrm{~Hz}$ from Eq. (1), which is very close to the measured frequency of excitation at $740 \mathrm{~Hz}$. Here, $l_{x}$ and $l_{y}$ are the longitudinal duct length and the duct width, respectively.

$$
\begin{gathered}
f=\frac{c}{2} \sqrt{\left(\frac{l}{l_{x}}\right)^{2}+\left(\frac{m}{l_{y}}\right)^{2}}=\frac{337.55312}{2} \sqrt{\left(\frac{1}{1.42}\right)^{2}+\left(\frac{1}{0.234}\right)^{2}}=730.994 \\
c_{e}=\frac{c}{\sqrt{1+\sigma}} \text { (2) }
\end{gathered}
$$

Here, $\sigma$ is the filling factor, $c$ is the sound speed $(340 \mathrm{~m} / \mathrm{s})$, and $c_{e}$ is the effective sound velocity, which is given by the Equation (2) [18]. The filling factor, which is given by the Equation (3), is the ratio occupied by the tube bank with respect to the whole domain in the duct, and as follows with considering the length $1420 \mathrm{~mm}$ shown in Fig. 2(a).

$$
\sigma=\frac{\text { Volume of tubes }}{\text { Volume of the duct }}=\frac{\left.9 * 19 * \pi \pi^{*} 6 \mathrm{~mm} / 2\right)^{\wedge} 2}{1420 \mathrm{~mm} * 200 \mathrm{~mm} * 234 \mathrm{~mm}}=0.0145551
$$

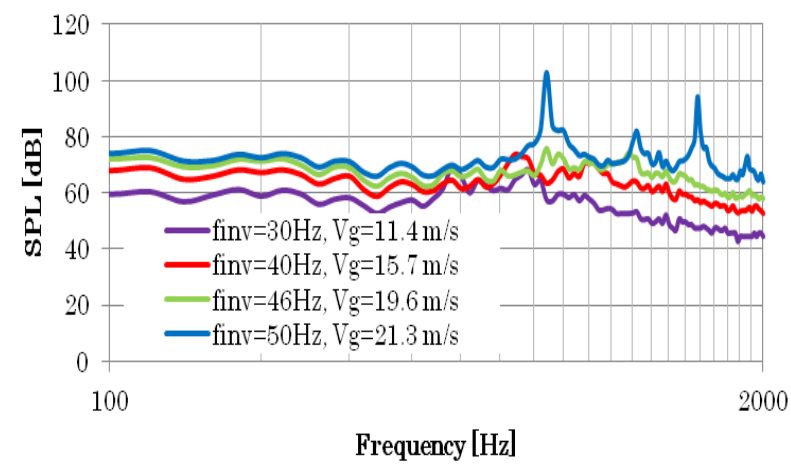

Fig. 3 Spectra of sound pressure level 


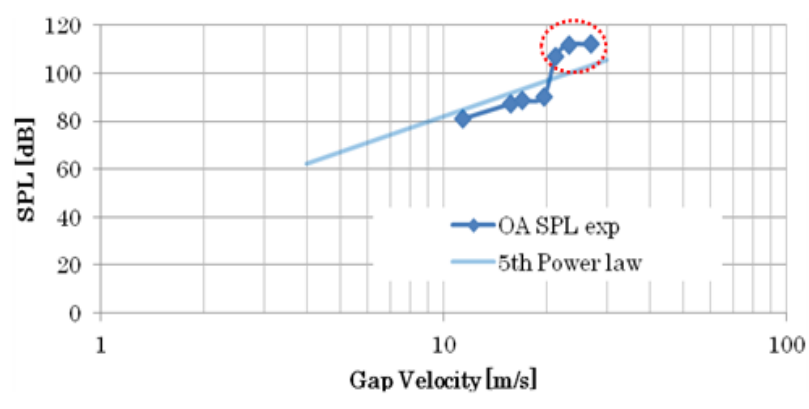

Fig. 4 Relation between overall sound pressure level $(200-2000 \mathrm{~Hz})$ and gap velocity

\section{NUMERICAL SIMULATIONS}

\section{A. Acoustic Simulations}

The BEM solver in commercial acoustic simulation package, WAON, is used to solve the acoustic characteristics [19]. In a sound field that satisfies the three-dimensional Helmholtz equation, the Kirchhoff-Helmholtz integral equation [20] for sound pressure is described as follows with respect to a point $\mathrm{p}$ and an area $S$ of a surface on a boundary.

$$
\frac{1}{2} \mathrm{p}\left(\mathbf{r}_{p}\right)=\int_{\Gamma}\left(p\left(\mathbf{r}_{q}\right) \frac{\partial G\left(\mathbf{r}_{p}, \mathbf{r}_{q}\right)}{\partial n_{q}}-\frac{\partial p\left(\mathbf{r}_{q}\right)}{\partial n_{q}} G\left(\mathbf{r}_{p}, \mathbf{r}_{q}\right)\right) d S+p_{d}\left(\mathbf{r}_{q}\right)
$$

In this solver, the following simultaneous linear equation, which is discretized from the Kirchhoff-Helmholtz integral equation for sound pressure (4), is solved:

$$
(\mathbf{E}+\mathbf{B}+\mathbf{C}) \mathbf{p}=\mathrm{j} \omega \rho \mathbf{A v}+\mathbf{p}_{\mathrm{d}}
$$

Here, $\mathbf{p}$ is the acoustic pressure vector, $\mathbf{v}$ is the particle velocity vector and the entries of the influence coefficient matrices are represented as follows:

$$
\begin{gathered}
\mathrm{E}_{\mathrm{ij}}=\frac{1}{2} \delta_{\mathrm{ij}},(6) \\
A_{i j}=\int_{\Gamma_{v}} N_{j}\left(\mathbf{r}_{q}\right) G\left(\mathbf{r}_{i}, \mathbf{r}_{q}\right) d S_{q},(7) \\
B_{i j}=\int_{\Gamma} N_{j}\left(\mathbf{r}_{q}\right) \frac{\partial G\left(\mathbf{r}_{i}, \mathbf{r}_{q}\right)}{\partial n_{q}} d S_{q},(8) \\
C_{i j}=\frac{j k}{Z_{j}} \int_{\Gamma_{A}} N_{j}\left(\mathbf{r}_{q}\right) G\left(\mathbf{r}_{i}, \mathbf{r}_{q}\right) d S_{q},(9) \\
G=\frac{\exp \left(j k\left|\mathbf{r}_{p}-\mathbf{r}_{q}\right|\right)}{4 \pi\left|\mathbf{r}_{p}-\mathbf{r}_{q}\right|},(10)
\end{gathered}
$$

where $\delta_{i j}$ is Kronecker delta, and $\Gamma$ is the total boundary. $\Gamma_{v}$ is a part of $\Gamma$ where the surface is assumed to be vibrating. $\Gamma_{A}$ is also a part of $\Gamma$ where the surface absorbs acoustic wave. $\mathbf{r}_{i}$ is a position vector of the $i$-th node, $\mathbf{r}_{p}$ is a

position vector of the monitor point $\mathrm{p}, \mathbf{r}_{q}$ is a position vector of the source point q, and $N_{j}$ is the interpolation function of the $j$-th node. $S_{q}$ is an area of elements in which the point q is included. $G$ is the fundamental solution of a three dimensional sound field. With the number of nodes $N$, the component $p$ of the vector $\mathbf{p}$ is as follows:

$$
p\left(\mathbf{r}_{q}\right)=\sum_{j=1}^{N} N_{j}\left(\mathbf{r}_{q}\right) p_{j},
$$

The component $p_{d}$ of the vector $\mathbf{p}_{d}$ is the direct pressure contribution from the acoustic source. Fig. 5 shows the boundary elements. There are 80,530 boundary elements. The origin of the Cartesian coordinate is placed at the center of the inflow boundary. To concern acoustic waves moving from the inflow boundary of the tube bank duct, a specific impedance boundary condition is imposed at the inflow boundary, whose value is $\rho c$, where $\rho$ is the density and $1.225 \mathrm{~kg} / \mathrm{m} 3$, and $c$ is the sound speed and $340 \mathrm{~m} / \mathrm{s}$. The outflow boundary is the surface connecting the duct inside and outside. At the outflow boundary, the interface boundary, where the particle velocity and acoustic pressure of the internal and external sound fields of the duct are coupled, is imposed. To clarify the acoustic characteristics of the tube bank duct, the acoustic frequency responses have been calculated using the monopole point sources (without the flow) shown in Fig. 5(b). The magnitude of the point sources is $1 \mathrm{~Pa}$ in all frequencies. The point source is located at $(0.5 H,-3.885 H, 0)$ on the downstream side of the tube bank, where $H$ is $200 \mathrm{~mm}$ in the height of the tube bank duct. In the self-sustained tone phenomenon with the flow, the sound source is supposed to be vortices generated on the downstream side behind the tube. Therefore, the sound source was placed on the downstream side of the tube bank also in the acoustic simulation. Furthermore, in order to excite the resonance mode in the duct width direction, the sound source was arranged asymmetrically with respect to the YZ plane.

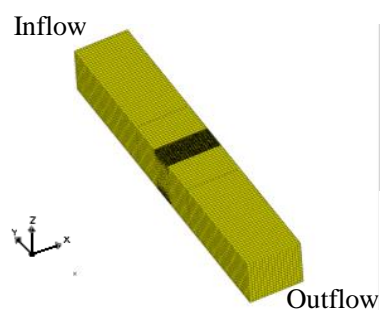

(a) Boundary element mesh Fig. 5 Boundary element mesh and monopole point source

\section{B. Unsteady CFD Simulations}

Unsteady flow fields in the duct are simulated in this paper. Inflow velocities are $\mathrm{U}=5.846,7.026,8.051,8.564,9.590$, 10.923 and $13.846 \mathrm{~m} / \mathrm{s}$, and corresponds to the gap velocities, $V_{g}=1.95 U=11.4,13.7,15.7,16.7,18.7,21.3$ and $27.0 \mathrm{~m} / \mathrm{s}$, respectively. Reynolds numbers, $R e_{D}$, based on the gap velocity, $V_{g}$, is prescribed to be in the range of 4,600 to 10,800 . The model for the CFD simulation is shown in Fig. 6. For these simulations, a three-dimensional computational domain in the duct has been applied. Unsteady flow fields are calculated with the commercial CFD code, ANSYS Fluent 
version 16.0, using an implicit compressible solver of second-order numerical accuracy in both space and time and its compressible LES (Dynamic Smagorinsky model) calculation features. The domain contains 4,944,100 cells and $5,156,304$ nodes. The cell spacing adjacent to the wall is $0.0003 \mathrm{~m}$. A steady velocity and temperature $(300 \mathrm{~K})$ conditions are imposed on the inflow boundary. Zero pressure outflow and non-reflective boundary conditions are applied on the outflow boundary. No-slip and adiabatic conditions are applied on the other walls. Steady state simulations were performed using Spalart-Allmaras (S-A) turbulence model and then used as initial conditions of transient LES simulations. The unsteady simulations were performed for 10000 time steps with a time step size $d t=1 e-5 s$, which corresponds to the non-dimensional time step based on $f_{v}$, 0.00733 .

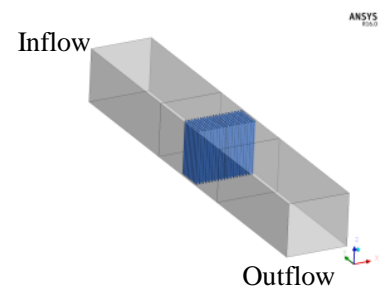

Fig. 6 Computational domain for CFD simulation

\section{Extraction of Acoustic Pressure on the duct wall}

Generally, it is difficult to solve an aerodynamic noise radiation to a far field through a direct solution of a compressible Navier-Stokes equation, because it requires a high accuracy scheme in both space and time, a large computational domain which contains a noise source and propagation regions, a fine mesh enough to resolve a far field sound pressure fluctuation and a high computational cost [21]. Therefore, a hybrid approach in which the flow field (the noise source) and the acoustic propagation are solved separately, are generally used to calculate the aerodynamic noise radiation to the far field [19]. However, in this paper, the interaction between the flow and acoustic fields are need to be solved when the resonance or self-sustained tone occurs, a high level sound is generated and the monitor point is near the noise source region. Therefore, the acoustic pressure is directly extracted from the unsteady compressible CFD simulations.

To convert the acoustic pressure time histories obtained from the CFD simulations into the frequency spectra, the discrete Fourier transform (DFT) has been applied. The acoustic pressure are extracted from 2500 steps (from $t=0.05$ $\mathrm{s}$ to $0.1 \mathrm{~s})$. The sampling period is $2 * d t=2 e-5 \mathrm{~s}$.

\section{RESUlTS AND DISCUSSION}

\section{A. Acoustic Simulations}

To clarify the acoustic characteristic of the tube bank duct, the acoustic frequency responses have been calculated using the monopole point source (without the flow) shown in Fig. 7. The monitor point is located at $(0.585 \mathrm{H},-4.76 \mathrm{H}, 0)$, that is on the downstream side of the tube bank and on the duct wall. Since, focused on the resonance frequency in the duct width direction, the sound pressure was measured on the duct wall where the sound pressure is assumed to be the largest. The peak frequency surrounded by the red circle is $720 \mathrm{~Hz}$, and close to the resonance frequency in the width direction of the duct, as mentioned in II.D. The peak frequency surrounded by the green circle is $1420 \mathrm{~Hz}$, and is close to a higher harmonics of the resonance frequency in the width direction. The peak frequency surrounded by the black circle is $1100 \mathrm{~Hz}$, and close to the coupled mode in the width, height and longitudinal directions, which is defined as follows.

$$
f=\frac{c_{e}}{2} \sqrt{\left(\frac{l}{l_{x}}\right)^{2}+\left(\frac{m}{l_{y}}\right)^{2}+\left(\frac{n}{l_{z}}\right)^{2}}=\frac{337.55312}{2} \sqrt{\left(\frac{1}{1.42}\right)^{2}+\left(\frac{1}{0.234}\right)^{2}+\left(\frac{1}{0.200}\right)^{2}}=1116.464
$$

Here, $l_{z}$ is the duct height. Fig. 8 shows acoustic modes on the wall of the duct. The acoustic mode in the width direction of the duct, its high-harmonic mode and the coupled mode in the width, height and longitudinal directions appear at $720 \mathrm{~Hz}$, $1420 \mathrm{~Hz}$, and $1100 \mathrm{~Hz}$, respectively. The acoustic mode at $720 \mathrm{~Hz}$ is dominant among the other acoustic modes and corresponds to the resonance frequency $\left(f_{a}=721.26 \mathrm{~Hz}\right.$, as mentioned in II.D) in the width direction of the duct rather than the coupled mode in the width and longitudinal directions. Thus, in the 3D acoustic simulation, the resonance frequency has been calculated, and clearly visualized.

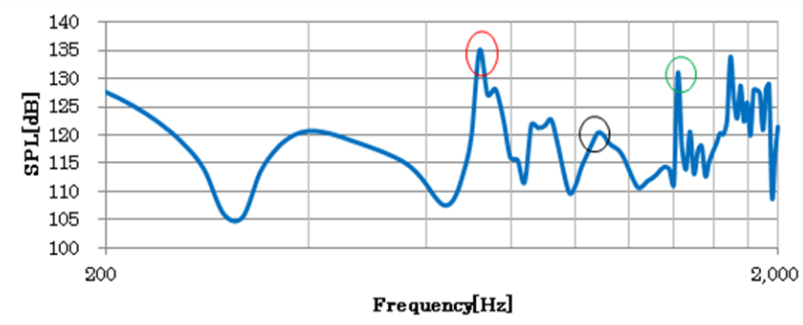

Fig. 7 Acoustic characteristic of the duct

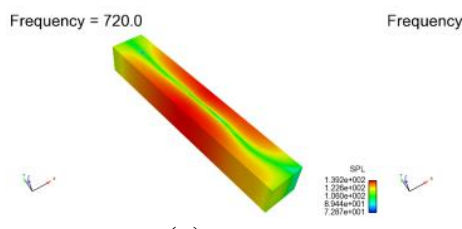

(a)

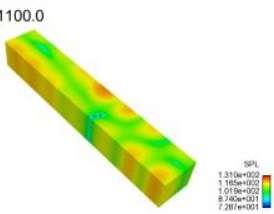

(b)

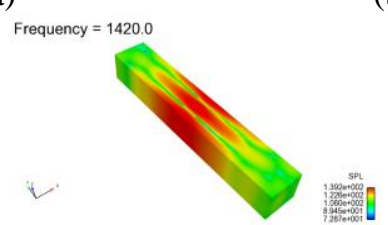

(c)

Fig. 8 Acoustic modes on the wall of the duct

\section{B. Unsteady CFD Results}

Instantaneous snapshots of vorticity fields at $\mathrm{Z}=0$ plane are shown in Fig. 9. Vortices generated around tubes travels downstream and form a vortex street. In the case of $V_{g}=21.3$ $\mathrm{m} / \mathrm{s}$, vortices are stronger than in the case of $V_{g}=11.4 \mathrm{~m} / \mathrm{s}$. Fig. 10 shows instantaneous snapshots of static pressure fields. As show in Fig. 10, the static pressure on the upstream side of the tube bank is larger than that on the downstream side. However, it is difficult to distinguish from the sound pressure with the static pressure. Therefore, the fluctuation pressure is defined as follows [21].

$$
d p=p_{s}-p_{\text {mean }}
$$


Here, $p_{s}$ is the static pressure which is defined by $p_{s}=p-p_{0}$ and $p_{\text {mean }}$ is the time-averaged of the pressure. The symbol $p_{0}$ denotes the ambient pressure. Fig. 11 shows instantaneous snapshots of a fluctuation pressure field at $Z=0$ plane. Comparing the cases of $V_{g}=21.3 \mathrm{~m} / \mathrm{s}$ with $V_{g}=11.4$ $\mathrm{m} / \mathrm{s}$, the fluctuation pressure in the case of $V_{g}=21.3 \mathrm{~m} / \mathrm{s}$ is much larger than in the case of $V_{g}=11.4 \mathrm{~m} / \mathrm{s}$. In the case of $V_{g}$ $=21.3 \mathrm{~m} / \mathrm{s}$, the pressure fluctuation mode in the width direction of the duct clearly appears.

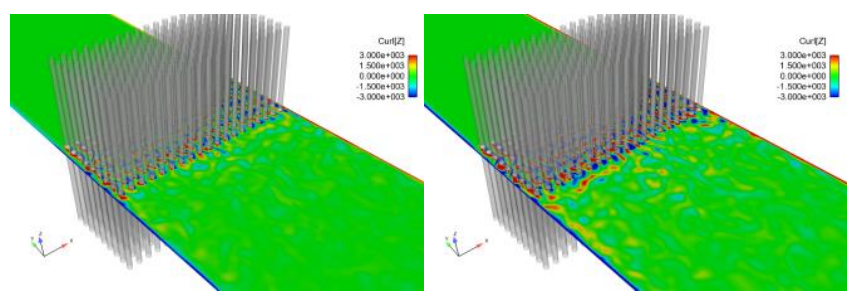

(a) $V_{g}=11.4 \mathrm{~m} / \mathrm{s}$

Fig. 9 Vorticity fields

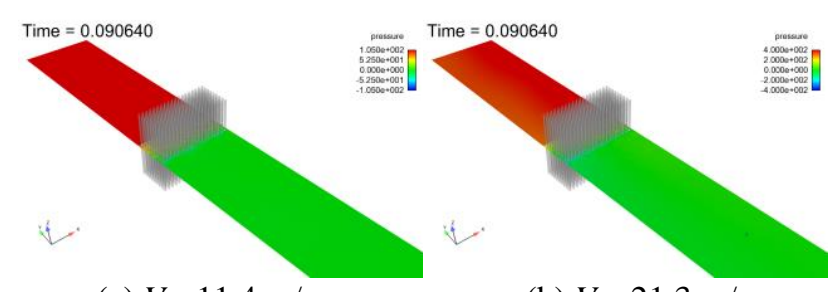

(a) $V_{g}=11.4 \mathrm{~m} / \mathrm{s}$

(b) $V_{g}=21.3 \mathrm{~m} / \mathrm{s}$

Fig. 10 Static pressure fields

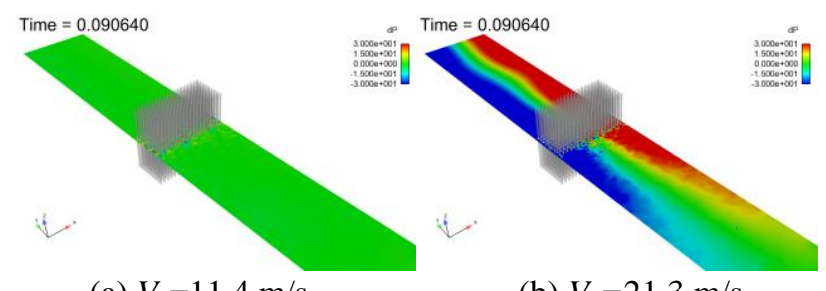

(a) $V_{g}=11.4 \mathrm{~m} / \mathrm{s}$

(b) $V_{g}=21.3 \mathrm{~m} / \mathrm{s}$

Fig. 11 Fluctuation pressure fields

\section{Sound Pressure Level from CFD Results}

Fig. 12 shows the frequency spectra of SPL on the wall of the duct near the outflow boundary. Both the simulated and measured data are shown in Fig. 12. In both simulations and experiments, the self-sustained tone occurs in the case of $V_{g}=$ $21.3 \mathrm{~m} / \mathrm{s}$, and the self-sustained tone does not occur in the case of $V_{g}=11.4 \mathrm{~m} / \mathrm{s}$. In the case of $V_{g}=21.3 \mathrm{~m} / \mathrm{s}$, the peak of self-sustained tone appears at about $740 \mathrm{~Hz}$ (surrounded by the red circle) and its high harmonic frequency, $1480 \mathrm{~Hz}$ surrounded by the green circle). This frequency is close to the resonance frequency in the duct width direction, $721.26 \mathrm{~Hz}$ and the resonance frequency simulated in the acoustic simulation, $720 \mathrm{~Hz}$, as mentioned in II.D and IV.A. The predicted SPL of the dominant tone at $740 \mathrm{~Hz}$ reasonably agrees with the measured one, and is assumed to contribute most to the overall SPL when the self-sustained tone occurs. Fig. 13 shows the relation between overall sound pressure level and the gap velocity in both simulations and experiments In the simulations as well as the experiments, the sound pressure level increases with increasing the gap velocity and follows the 5th power law when the gap velocity is low.
However, the sound pressure level does not follow the 5th power law when the gap velocity is high (points surrounded by the red circle), and remains high with increasing the gap velocity. As shown in Figs. 12 and 13, the simulations show a reasonable agreement with the experiments in terms of the generation prediction of the self-sustained tone.

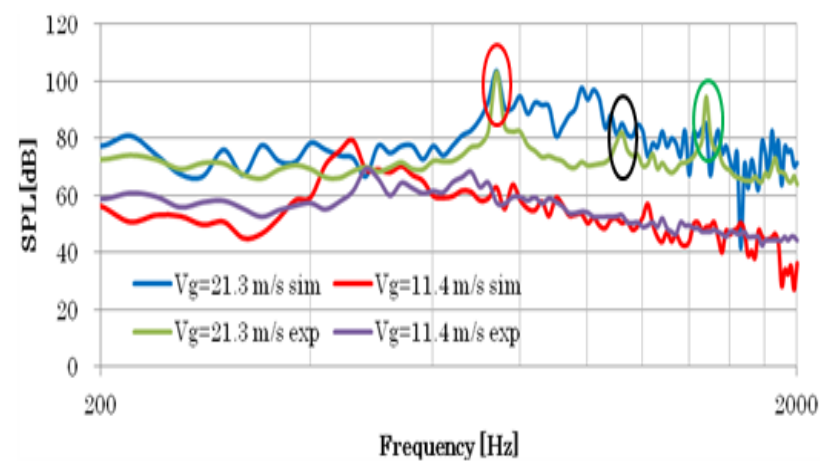

Fig. 12 Spectra of sound pressure level

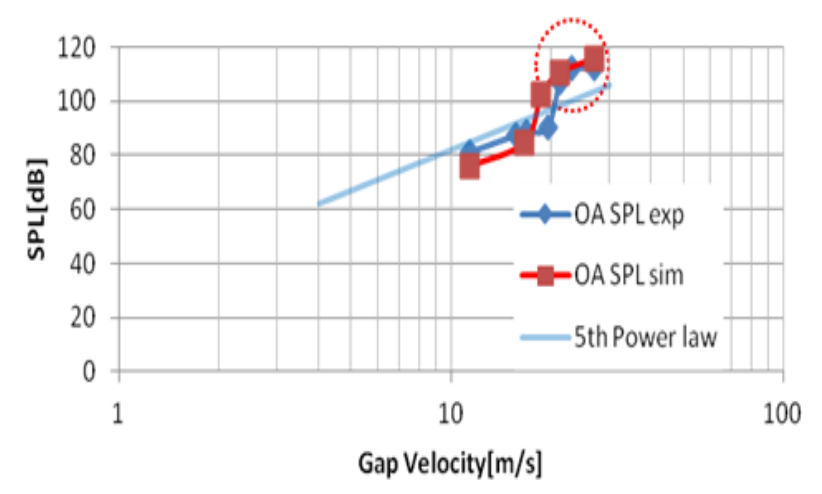

Fig. 13 Relation between overall sound pressure level $(200-2000 \mathrm{~Hz})$ and gap velocity

Fig. 14 shows SPL on the wall of the duct in frequency domain. In CFD simulations, as shown in Fig. 14, the acoustic modes does not appear in the case of $V_{g}=11.4 \mathrm{~m} / \mathrm{s}$, however, those clearly appears in the case of $V_{g}=21.3 \mathrm{~m} / \mathrm{s}$, as in the experiments. Fig. 14(d) shows that the acoustic mode in the width direction or the coupled mode in the width and longitudinal directions appears at $740 \mathrm{~Hz}$ in the case of $V_{g}=$ $21.3 \mathrm{~m} / \mathrm{s}$. The acoustic mode at $740 \mathrm{~Hz}$ shown in Fig. 14(d) corresponds to the fluctuation pressure field in Fig. 11(b). Accordingly, the acoustic mode in the width direction or the coupled mode in the width and longitudinal directions is dominant in the self-sustained tone. The acoustic mode at $1480 \mathrm{~Hz}$ is a high-harmonic mode of that at $740 \mathrm{~Hz}$, and clearly appears in the case of $V_{g}=21.3 \mathrm{~m} / \mathrm{s}$, as shown in Fig. 14(f). On the other hand, the acoustic mode at $1120 \mathrm{~Hz}$ in Fig. $14(\mathrm{e})$ is the coupled mode in the width, height and longitudinal directions defined by equation (12). This mode also appears in both CFD simulations and the experiments (peak surrounded by the black circle), as shown in Fig. 12. These modes are close to the acoustic modes obtained from the acoustic simulation and the experiments. It can be presumed that the slight difference in the resonance frequencies obtained from the acoustic simulation (without the flow) and the CFD simulation or the experiments is due to the absence or presence of the flow. This is because the resonance frequency slightly increases with increasing the gap 
flow velocity, as shown in the Fig. 1. Furthermore, the acoustic mode at $740 \mathrm{~Hz}$ is dominant among the other acoustic modes and corresponds to the resonance frequency $\left(f_{a}=721.26 \mathrm{~Hz}\right.$, as mentioned in II.D) in the width direction of the duct rather than the coupled mode in the width and longitudinal directions.

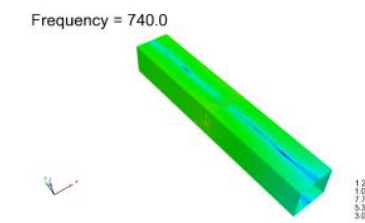

(a) $V_{g}=11.4 \mathrm{~m} / \mathrm{s}$
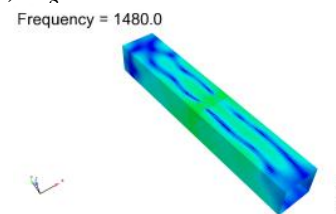

(c) $V_{g}=11.4 \mathrm{~m} / \mathrm{s}$

Frequency $=1120.0$

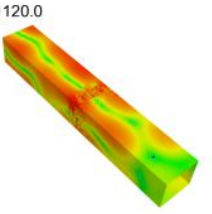

(d) $V_{g}=21.3 \mathrm{~m} / \mathrm{s}$

Fig. 14 SPL on the wall of the duct

\section{CONCLUSION}

In this paper, we have performed numerical simulations of high level sound generated in the boiler tube bank duct with the flow and the acoustic simulation without the flow using commercial CFD and an acoustic BEM simulation codes and compared the simulation results with the measurements. The following concluding remarks are obtained in this paper.

1) In the acoustic simulation without the flow, the typical acoustic modes in the duct have been calculated using the point source which was placed on the downstream side of the tube bank. They correspond to the acoustic mode in the width direction of the duct, its high harmonic mode, and the coupled mode in the width, height and longitudinal directions. The acoustic mode in the duct width direction is dominant among the other acoustic modes.

2) CFD simulations show a reasonable agreement with the experiments in terms of the generation prediction of the self-sustained tone. In CFD simulations, the sound pressure level increases with increasing the gap flow velocity and follows the 5th power law when the gap velocity is low. However, the sound pressure level does not follow the 5th power law when the gap velocity is high, as in the experiments.

3) The acoustic modes obtained from the acoustic simulation correspond to those obtained from the CFD simulation and the experiments. However, there is the slight difference in the resonance frequencies obtained from the acoustic simulation (without the flow) and the CFD simulation or the experiments in that the resonance frequency slightly increases with increasing the gap flow velocity.

\section{REFERENCES}

[1] R.C. Baird, "Pulsation-Induced Vibration in Utility Steam Generation Unit," Combust., vol. 25, 1954, pp. 38-44

[2] P.R. Owen, "Buffeting Excitation of Boiler Tube Vibration," J. Mech. Eng. Sci., vol. 7, iss. 4, 1965, pp. 431-439.

[3] Y.N. Chen, "Flow-induced Vibration and Noise in Tube-bank Heat Exchangers due to Von Karman Streets," J. Eng. Ind., vol. 90, iss. 1, 1968, pp. 134-146.

[4] M. Funakawa, "The Acoustic Resonance in a Tube Bank," J. JSME., vol. 13,1970 , pp. 348-355.

[5] J.A. Fitzpatric, "The Prediction of Flow-Induced Noise in Heat Exchanger Tube Arrays," J. Sound. Vib., vol. 99, 1985, pp. 425-435.

[6] R.D. Blevins, "Bressler M.M. Acoustic Resonance in Heat Exchanger Tube Bundles-Part I: Physical Nature of the Phenomena," J. Press. Vess. Technol., vol. 10, 1987, pp. 275-281.

[7] D.S. Weaver, "Vortex Shedding and Acoustic Resonance In Heat Exchanger Tube Arrays," Technology for the 90's, ASME Special Publication, New York, 1993; pp.776-810.

[8] S. Ziada and A. Oengören, "Vortex Shedding in an In-Line Tube Bundle with Large Tube Spacings," J. Fluid. Struct., vol. 7, iss. 6, 1993, pp. 661-687.

[9] S. Ziada, "Vorticity Shedding and Acoustic Resonance of Tube Bundles,” J. Braz. Soc. Mech. Sci., vol. 28, No. 2, 2006, pp. 186-199.

[10] F. L. Eisinger and R. E. Sullivan, "Acoustic Resonance in a Package Boiler and Its Solution A Case Study," J. Press. Vess. Technol., vol. 129, 2006, pp. 759-762.

[11] H. Hamakawa, H. Matsue, E, Nishida and T. Fukano, "Acoustic Resonance and Vortex Shedding from Tube Banks of Boiler Plant," $J$. Fluid. Sci. Technol., vol. 3, no. 6, 2008, pp. 805-813.

[12] H. Hamakawa, T, Nakamura, K, Asakura, E. Nishida and E. Kurihara, "Effect of Arrangement of Tube Banks on Acoustic Resonance," O. J. Fluid. Dy. vol. 2, 2012, pp. 311-317.

[13] K. Ishihara, T. Tamehira, M. Tsujii and M. Ichimiya, "Study on a Countermeasure of Self-Sustained Tone by a Baffle Plate in Boiler Tube Banks," J. Basic. Appl. Phys., vol. 2, iss. 3, 2013, pp. 148-154.

[14] J. Kim, B. Cho, K. Kim, M.Y. Ha, C. Son and S.Y. Yoon, "Acoustic Resonance and Pressure Drop through a Staggered Tube Bundle in a Compact Heat Exchanger," Appl. Acoust., vol. 91, 2015, pp. 63-67.

[15] K. Ishihara and M. Nakaoka, "Study on a Countermeasure Using Walls Made of Perforated Plate for High Level Sound," In proceedings of ASME 2015 Pressure Vessels and Piping Conference Volume 4: Fluid-Structure Interaction, Boston, Massachusetts, USA, 19-23, July, 2015.

[16] R. Hanson and S. Ziada, "Effect of acoustic resonance on the dynamic lift of tube arrays," J. Fluid. Struct., vol. 27, 2011, pp. 367-382.

[17] A. Mohany, D. Arthurs, M. Boldu, M. Hassan and S. Ziada "Numerical and experimental investigation of flow-acoustic resonance of side-by-side cylinders in a duct," J. Fluid. Struct., vol. 48, 2014, pp. 316-331.

[18] K. Ishihara, M. Miyamoto, Y. Yoshida, "Study on the Evaluation of Sound Speed in Duct with Tube Banks," J. Environ. Eng., vol. 5, no. 2, 2010, pp. 339-352.

[19] M. Mori, T. Masumoto and K. Ishihara, Study on Acoustic, "Vibration and Flow Induced Noise Characteristics of T-shaped Pipe with a Square Cross-Section," Appl. Acoust., vol. 120, 2017, pp. 137-147.

[20] A. D. Pierce, Acoustics: an introduction to its physical principles and applications. McGraw-Hill, New York, 1981.

[21] O. Inoue and N. Hatakeyama, "Sound generation by a two-dimensional circular cylinder in a uniform flow," J. Fluid Mech., vol. 471, 285, 2002, pp. 285-314. 